\title{
Nonlinear Nonlocal Algorithm for Video Filtering
}

\author{
Ahmed Fouad El Ouafdi ${ }^{* *}$, Hassan El Houari ${ }^{2}$ \\ ${ }^{1}$ Labsiv Laboratory, Department of Computer Sciences, Faculty of Sciences, Ibn Zohr University, Agadir, \\ Morocco \\ 2 LaGuardia Community College, CUNY, New York, USA \\ * Corresponding author's e-mail: eouafdi@gmail.com
}

\begin{abstract}
Video sequences are frequently contaminated by noise throughout the acquisition process, resulting in considerable degradation of video display quality. In this paper, we present a novel method of video filtering. The proposed filter is developed from an optimization problem in which a Bayesian term and a noisy video sequence prior distribution are combined. The method begins by segmenting the video sequence into space-time blocks and then substituting each noisy block by a weighted average of non-local neighbor blocks. Gradient-based weights are used to dynamically adjust the edge preservation and smoothness of the reference block. The obtained formulation enables nonlinear filtering and, hence, preserving key features such as edges and corners while using the intrinsic Bayesian filtering framework. Experiments on different video sequences with varying degrees of noise show that the proposed method performs better than state-of-the-art video filtering approaches.
\end{abstract}

Keywords: video filter; noise reduction; nonlocal filter; bayesian filtering; nonlinear filter.

\section{INTRODUCTION}

Throughout the acquisition process, video sequences are often contaminated by noise, degrading the picture and video display quality significantly and adversely affecting post-processing performance in such as motion tracking, object detection, feature extraction, and pattern recognition. Numerous noise reduction techniques have been published in recent years, and the majority of them can be classified into two broad categories based on whether the noise reduction process by exploiting spatial as well as temporal correlations between the frames [1], or by employing nonlinear diffusion techniques to boost edges while the noise is reduced [2]. In what follows, we highlight the most important methods from each category.

From the first category, Yan et al. [3] propose a temporal filtering technique based on extracting and modeling noise in continuous frames of video sequences. The filter was shown to be effective in reducing noise in real-time video sequences, but suffers from dragging effects on moving objects. Another filter based on temporal data blocks is presented in [4], the filter is efficient at reducing noise while minimizing blocking artifacts, however, it does not prevent the formation of blurred edges. Due to the fact that both spatial and temporal filters may produce blurring in motion regions independently, the concept of considering both space and time information in order to avoid temporal artifacts is raised. For example, [5] presents a method for reducing video noise based on the space-time correlations between adjacent frames; to get an appropriate results, Kalman and bilateral filtering were integrated into the filter. Another method, based on spatial-temporal combination, is presented in [6]. It discriminates between static regions and the motion of video sequences' regions. The authors of [7] proposed a technique for minimizing noise in videos that have been affected by random and fixed-pattern noise by using motion-compensated 3D space-time volumes. 
To enhance filtering, especially in the large-scale TV noise region, [27] developed an adaptive filter that adapts temporal and spatial characteristics in real time. The authors of [28] suggest a recursive noise reduction filter for video based on nonlocal means that allows filtering for temporary correlations.

The second category of video sequence filters uses nonlinear diffusion techniques to increase the sharpness of edges while reducing noise [11, 12]. To guarantee numerical stability, this kind of filter discretizes partial differential equations using a finite difference together with a semi-implicit temporal discretization schema. In this setting, an improvement to the nonlinear smoothing method for minimizing video noise and enabling a more efficient compression operation is proposed [14]. In [11] and [12], the space-time diffusion tensor is used to improve each video frame in the intensity change principal direction. While these nonlinear techniques provide pleasing visual effects and increase the quantity of video sequences $[15,16]$, they suffer from blurring critical video sequence characteristics such as edges and corners. To overcome this limitation, [17] implements an isotropic diffusion equation using a finite difference technique to improve video sequence patterns in terms of optical flow computation. Similarly, [18] presents an anisotropic diffusion technique for real-time video noise reduction; this method replaces the Gaussian diffusion kernel with a median mean value in order to improve the image's quality while decreasing the filter's processing time through GPU optimization.

To our understanding, the best video filtering results are currently achieved using nonlocal Bayesian patch methods. In this context, [7] and [10] present a Bayesian 3D patch video filter based on space-time rectangular patches. Each patch, is filtered using the maximum a posterior estimator, which is considered as a sample of the Gaussian distribution. Another rapidly growing family of video filtering techniques, such as DnCNN, is based on convolutional neural networks (CNN) [19, 20]. Recently, nonlocal network for noise reduction in video sequences was suggested [16]. The network is constructed by combining CNN with a method known as self-similarity searchm. The objective is to determine the most comparable patches for each patch through its initial non-trainable layer, and then utilize this information to forecast the clean video sequence using CNN.

\section{CONTRIBUTION}

Although the non-local patch-based methods are state-of-the-art video filtering, they have the drawback of blurring important video sequence features. To address this though non-local patchbased techniques are state-of-the-art methods for video filtering, they have the disadvantage of blurring critical video sequence features. To address this drawback, we propose solving an optimization problem that incorporates both a posterior Bayesian term and prior probability distribution of the observed noisy video sequence. The derived noise reduction formula then incorporates a nonlinear diffusion component into the Bayesian expression, increasing filtering on the homogeneous region and decreasing filtering on the video frame's local properties. This kind of filtering preserves video sequence's local features. To take advantage of the noise redundancy, we developed a Bayesian nonlinear filter on a block-wise nonlocal space-time region. This allows the usage of redundant information throughout the video sequence while still preserving important features.

\section{Bayesian Formulation}

The observed noisy video sequence is usually represented as a patch additive form:

$$
\tilde{u}=\mathrm{u}+\eta
$$

where: $u$ stands for the ideal (unknown) patch, and $\tilde{u}$ is the observed patch corrupted by the noise term $\eta$ modeled as an independent and identically distributed Gaussian distribution.

Bayesian methods are intensively used in the image filtering literature. Such approaches introduce prior knowledge and impose limitations in the estimation process. The Bayesian estimator, known in practice as the maximum a posterior (MAP) approach, is described as the maximum of the following posterior distribution:

$$
\arg \max _{u} P(u \mid \tilde{u})
$$

The Bayesian approach finds the patch $u$ that maximizes the posterior probability given the observed one $\tilde{u}$. By Bayes rule, we have:

$$
P(u \mid \tilde{u})=\frac{P(u) \cdot P(\tilde{u} \mid u)}{P(\tilde{u})}
$$

where: $\tilde{u}$ is the observed noisy patch. 
As the logarithm is a monotone function and the probability function $P$ is positive, an equivalent formulation of (2) using Bayes rule (3) leads to:

$$
\arg \max _{u}(\log (P(u)+\log (P(\tilde{u} \mid u)))
$$

Notice that the probability term $\mathrm{P}(\tilde{u})$ is omitted from the previous equation since it is independent to $u$. The above optimization problem is composed of the prior patch distribution $\mathrm{P}(u)$ and the posterior Bayesian patch distribution $\mathrm{P}(\tilde{u} / u)$. In what follows, we explicitly define each term in the optimization problem (4).

\section{The prior patch distribution}

In the case of linear model (1) with additive Gaussian noise, the patch prior $P(u)$ of $u$ is also a Gaussian distribution with covariance matrix and expectation patch $\tilde{u}$, which means that:

$$
P(u)=\frac{1}{A_{0}} \cdot \exp \left(-(u-\bar{u})^{T} C_{u}^{-1}(u-\bar{u})\right)
$$

where: $A_{0}$ is a coefficient of normalization.

In the next section we calculate the second component of (4) based on the posterior distributions.

\section{The posterior Bayesian patch distribution}

To compute the posterior Bayesian patch distribution, consider the video sequence as a dynamical diffusion framework of particles in a space-time domain, with each particle representing a pixel in the video sequence [22]. Then, according to stochastic diffusion theory [23, 24], the specific position of the particles at any given time period cannot be calculated precisely; instead, only the probability of the particle being located in a particular region area can be estimated. Specifically, the transfer probability density of the particle has the following form:

$$
P(\tilde{u} \mid u))=\frac{1}{A_{1}} \exp -\frac{(u-\tilde{u}) D^{-1}(u-\tilde{u})}{\sigma^{2}}
$$

where: $D$ is the diffusion diagonal matrix, and $A_{1}$ is a positive normalization coefficient.

\section{Nonlinear Bayesian filter formulation}

By substituting (5) and (6) in the optimization problem (4), we get the following equivalence of optimization problem argmin for each observed patch $u$ : $\arg \min _{u}\left((u-\bar{u})^{T} C_{u}^{-1}(u-\bar{u})+\frac{(u-\tilde{u}) D^{-1}(u-\tilde{u})}{\sigma^{2}}\right)$

Due to the fact that the noisy patch $\tilde{u}$ and the noise-free patch $u$ cannot be directly observed, we can only get an empirical estimate covariance matrix $C_{\tilde{u}}$ from (1). Assuming that the noise follows a Gaussian distribution with a standard deviation $\sigma$ so that:

$$
C_{\widetilde{u}}=C_{u}+\sigma^{2} \mathrm{I}
$$

The problem (7) may be reduced to a minimization problem by using the formula (8) as:

$\arg \min _{u}\left((u-\bar{u})^{T}\left(C_{\tilde{u}}-\sigma^{2} I\right)^{-1}(u-\bar{u})+\frac{(u-\tilde{u}) D^{-1}(u-\tilde{u})}{\sigma^{2}}\right)$

When the previous function is differentiated with respect to $u$ and equated to zero, the following result is obtained:

$\sigma^{2}\left(C_{\widetilde{u}}-\sigma^{2} I\right)^{-1}(u-\bar{u})+D^{-1}(u-\tilde{u})=0$

By rearranging the terms, we get:

$$
u=\tilde{u}+D\left[\sigma^{-2} C_{\tilde{u}}+D-I\right]^{-1}(\bar{u}-\tilde{u})
$$

as an optimal solution to the problem (7).

\section{Nonlocal Nonlinear Noise Reduction}

Patch-based video models may be thought of as three-dimensional (space-time) expansions of the conventional two-dimensional image block matching model. By including temporal information, we may take use of redundancy in motion information. As with the image non-local PatchBased algorithm [24, 25, 26], the proposed implementation consists of three-stages: a) identifying and grouping in a 4D block space-time volumetric patches that are similar to a reference patch; b) using a collaborative filter; and c) aggregating the collaborative filter, which is implemented in two steps: 1) Bayes' formula is applied to the 4D block, and 2) the 4D block is relocated. This 3D filtering is performed simultaneously on a group of 4D video blocks. Due to the overlap of the filtered patches, multiple estimates for each pixel need to be combined. Aggregation is a special process of averaging that makes use of this redundancy.

\section{Space-time patches grouping}

We begin by using the patch grouping method extensively used in the image processing literature for video filtering. Consider a noisy video 
sequence that has been evenly discretized along the time axis into a set of images.

With reference to Figure 1, assume that we want to filter the frame of the image sequence using the previous frame and the next frame, and let be the reference observed noisy patch to be filtered with size (seen as a column vector) of the noisy image .

Within a centred windows with size, we select the similar patch to the reference patch as

$$
\Delta\left(\tilde{u}_{k}, \tilde{Q}_{k}\right)=\frac{\left\|\tilde{u}_{k}-\tilde{Q}_{k}\right\|^{2}}{\left(k_{1}\right)^{2}}
$$

Where $\alpha$ is a threshold parameter and is the set formed by the $\mathrm{N}$ closest similar patches to the reference patch . The extension to the space-time patch grouping volume is performed as follows: for each similar patch, , we calculate the displacement vectors that identify the patch in the preceding and subsequent frames. The sequence is then explicitly filtered along the motion trajectories using motion estimation as a pre-processing step. As shown in Figure 1, we refer to the spacetime volume patch that is obtained by grouping all similar neighbor patches. The reference patch and all its similar patches with (10) are then restored using the covariance estimation (8) and the expectation block:

$$
\begin{aligned}
\overline{\tilde{u}}_{k} & =\frac{1}{N} \sum_{\tilde{Q}_{k} \in V\left(\tilde{u}_{k}\right)} \tilde{Q}_{k} \\
C_{\tilde{u}_{k}} & =\frac{1}{N} \sum_{\tilde{Q}_{k} \in V\left(\tilde{u}_{k}\right)}(\tilde{Q}-\bar{u})\left(\tilde{Q}_{k}-\overline{\tilde{u}}_{k}\right)^{T}
\end{aligned}
$$

Algorithms 1 describes the proposed video denoising technique.
Algorithm 1. Nonlinear nonlocal Bayes video filtering algorithm

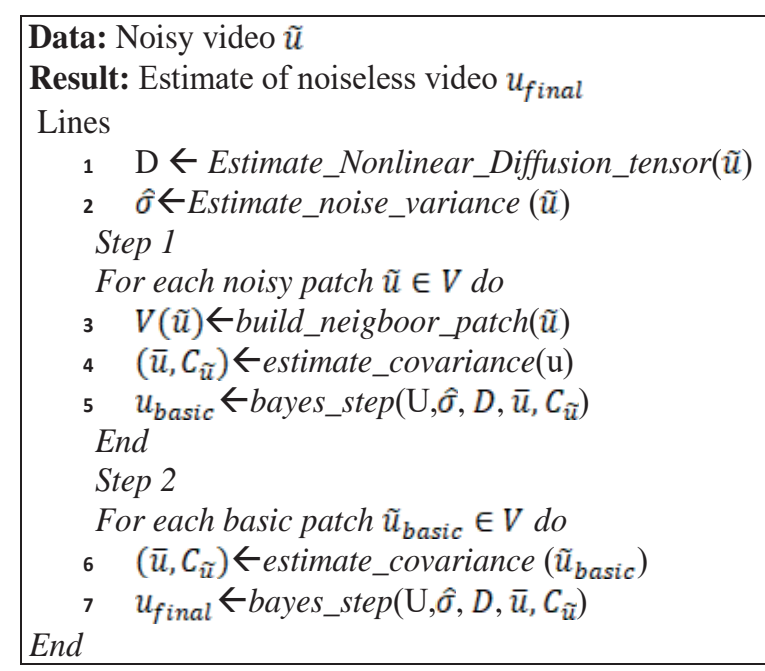

\section{Nonlinear filter parameters: local features preserving}

To begin, we estimate the diagonal diffusion matrix $D=\operatorname{diag}(, \cdots$,$) for the whole sequence,$ which enables to perform nonlinear diffusion, where $n$ is the size of the frame Each diagonal term of the diagonal diffusion matrix is given by

$$
d_{i}=\frac{1}{1+\lambda_{0}^{i}+\lambda_{1}^{i}+\lambda_{2}^{i}}
$$

where: $\lambda_{0}^{i}, \lambda_{1}^{i}$ and $\lambda_{2}^{i}$ are the eigenvalues of the space-time structure tensor which is defined at each point by:

$$
K_{\rho}=G_{\rho} \star\left(\nabla I_{k} \nabla I_{k}^{T}\right)
$$

where: $\nabla I_{k}=\left(\begin{array}{lll}\frac{\partial I_{k}}{\partial x} & \frac{\partial I_{k}}{\partial y} & \frac{\partial I_{k}}{\partial t}\end{array}\right)$ stands for the spacetime gradient operator.

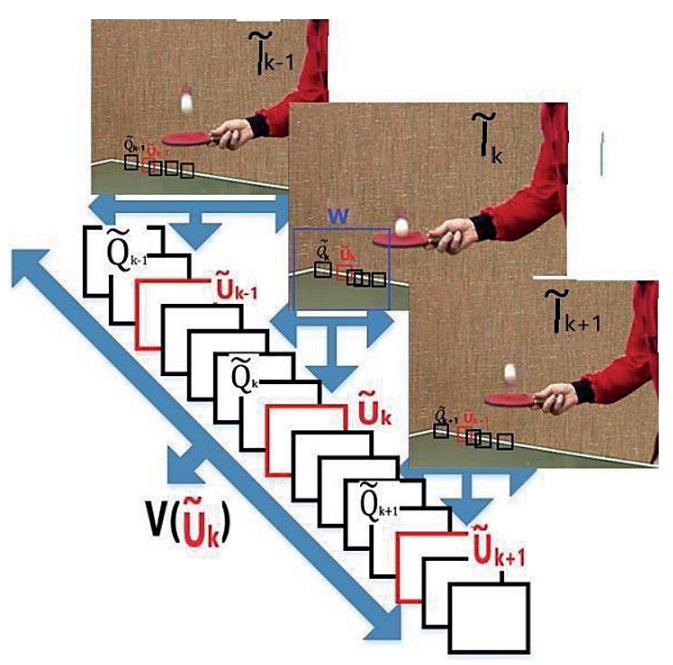

Fig. 1. Space-time grouping of similar neighbor patches 
For space-time homogeneous structure, all the eigenvalues are small $\approx 0$, in this case, $\mathrm{d} \approx 1$ and therefore, space-time isotropic filtering takes place. The situation in which moving a corner or edge structure corresponds to $\lambda_{1,2,3} \gg 1$, characterizes a shifting pixel on an edge or corner structure along the trajectory of motion. In this instance, the nonlinear filtering is performed, and hence the edges and corners are preserved throughout the filtering process.

As described in [16, 25, 26, 27] and [28], we reduce noise via two filtering stages. To begin, we group the space-time neighborhood of each noisy patch in line 3 using the patch distance (11) as illustrated in Figure 1. Following that, we use the equation (12) to estimate the mean and variance patches. By using the Bayes role stated in equation (10), we get a basic estimation and of the reference patch and all its similar patches in line 5. In the second stage, we calculate the final estimation patch and using the basic estimate as an oracle. That is, we construct the space-time volume by grouping all similar neighbor patches; the mean and covariance matrices are computed in line 6 , and the final noise-free patch is estimated in line 7 using patches from the basic estimates and .

\section{EXPERIMENTAL RESULTS}

In this section, the proposed non-linear nonlocal Bayes video filtering algorithm (NNBA) is evaluated on grayscale and color sequences. The first application compares the filtering outputs of three state-of-the-art methods using real grayscale video sequences that have been contaminated with artificial Gaussian noise. We calculate the PSNR (the Peak Signal to Noise Ratio) as an objective performance evaluation to quantify the visual difference between the processed and original video sequences. We consider the qualitative evaluation via visualization of the filtered video sequences. The standard video sequences such as "Tennis", "Flower Garden, "Salesman", and "Miss America" are used for the evaluation of the proposed filter. The sequences "Salesmam" and "Miss America" are characterized by fast variation and slow variation objects on stationary backgrounds. The "Tennis" sequence has fine textured regions, rotation motions, zooming, and panning, while the Flower Garde" contains a wide textured region and steady translation motion. We evaluate our filter on two color video sequences: the
"Flower Garde" sequence with additive Gaussian noise, and the lizard and TV sequences that are naturally contaminated with noise.

\section{Quantitative evaluation}

To begin filtering the noisy video sequences, we set the search window's dimension, the patch size , and the dimension of each pixel's spacetime neighborhood $\mathrm{N}$. To simplify the parameter selection procedure, we pick the set of parameters that provides the filter's highest mean PSNR when applied to noisy video sequences. Numerous tests have been conducted to fine-tune the suggested parameters, which have a major impact on the filtering efficiency as a whole. We utilize a single set of settings in these experiments: the values of the nearest comparable patches are set to $\mathrm{N}=21$, the size of the search window is set to $=21$, and the size of the reference patch is set to $=9$. As a consequence, the filtering results are enhanced. The motion field for the current video frame is estimated using the block matching method with a block size of 16 and a searching parameter of 7 . The Gaussian convolution parameter is set at in equation (14). That is, for each frame, we calculate the derivative gradient for each pixel inside a five-dimensional window.

To process each reference patch in the current frame, the filtering process involves both the previous and subsequent frames along the estimated motion trajectory, and at each patch center, a space-time neighborhood of dimension 21 is evaluated, as is shown in Figure 1. The proposed filter was compared to three known filters in the literature: the video block-matching filter (VBM4D) [29], the video network (DnCNN) [19], and the motion-adaptive space-time filter built on the $\mathrm{K}$-closest neighborhood (3DKNN) [30].

On conduct this comparison, we applied the proposed filter to noisy sequences and compared it to the other sequences filtered using the three comparative techniques. We examined the sequences accessible in the public domain to offer an impartial comparative evaluation.

In the first set of experiments, we introduced a modest quantity of artificial Gaussian noise with variances of $\sigma=15$ and $\sigma=20$ to the original video sequence, averaging PSNR. By providing the maximum PSNR for all evaluated sequences, the proposed filter outperforms the majority of comparable filters; we observe that the network DnCNN overfits motion patterns 
Table 1. Average of PSNR in term of noise levels

\begin{tabular}{|c|c|c|c|c|}
\hline Sequences & \multicolumn{2}{|c|}{ Salesman } & \multicolumn{2}{c|}{ Miss America } \\
\hline Noise $(\sigma)$ & $15 \mathrm{~dB}$ & $20 \mathrm{~dB}$ & $15 \mathrm{~dB}$ & $20 \mathrm{~dB}$ \\
\hline Algorithms & \multicolumn{3}{|c|}{} \\
\hline DnCNN & 31.27 & 29.52 & 34.95 & 31.87 \\
\hline 3DKNN & 30.20 & 29.56 & 35.98 & 32.91 \\
\hline VBM4D & 34.10 & 31.43 & 37.54 & 36.04 \\
\hline NNBA & 35.17 & 33.57 & 38.77 & 37.31 \\
\hline
\end{tabular}

throughout training and fails when confronted with a different motion. In general, the proposed filter performs optimally in terms of PSNR for almost all test video sequences. As shown in Table 1, the results are displayed in terms of the averaged PSNR. The proposed filter surpasses most other comparable filters by providing the maximum PSNR for all evaluated sequences. We notice that the network DnCNN over fit motion patterns in the training and fails when it encounters a different motion. In Figure 8, the graph depicts each frame's PSNR for the four processed sequences.

An expended view of the filtered frame is the addition of a wide textured region and steady translation motion. We evaluate our filter on two color video sequences: the" Flower Garden" sequence with additive Gaussian noise and the lizard and television sequences with naturally occurring noise.

\section{Quantitative evaluation}

Along with quantitative evaluation, we evaluate the visual quality of the proposed filter's output to that of the three filtering techniques. Since all filters are particularly effective at reducing low levels of noise, we purposefully compared the results for high-noise sequences with noise level $\sigma$ $=20$, and as illustrated in Figure 2, Figure 3, and Figure 4, the proposed filter results in a remarkable reduction of noise with less temporal and spatial blurring than other filters.

We use the proposed algorithm for filtering two color video sequences, one with natural noise and one with artificial Gaussian noise. In Figure 6, we illustrate a video sequence with artificial noise; as with the grey-level video sequences case, the visual evaluation shows that the proposed approach achieves significant results in terms of artificial noise removal. In Figure 7 , we display a video sequence with natural noise, which was processed by TV cameras. The camera introduced the noise. The results of noise reduction on the Lizard sequence are displayed, and on the filtered frames, we can see that the proposed filter can effectively reduce noise while preserving essential frame features, as shown in the expanded view of the image. Figure 9 illustrates the results of noise reduction on a television video sequence, demonstrating that noise reduction is successful.
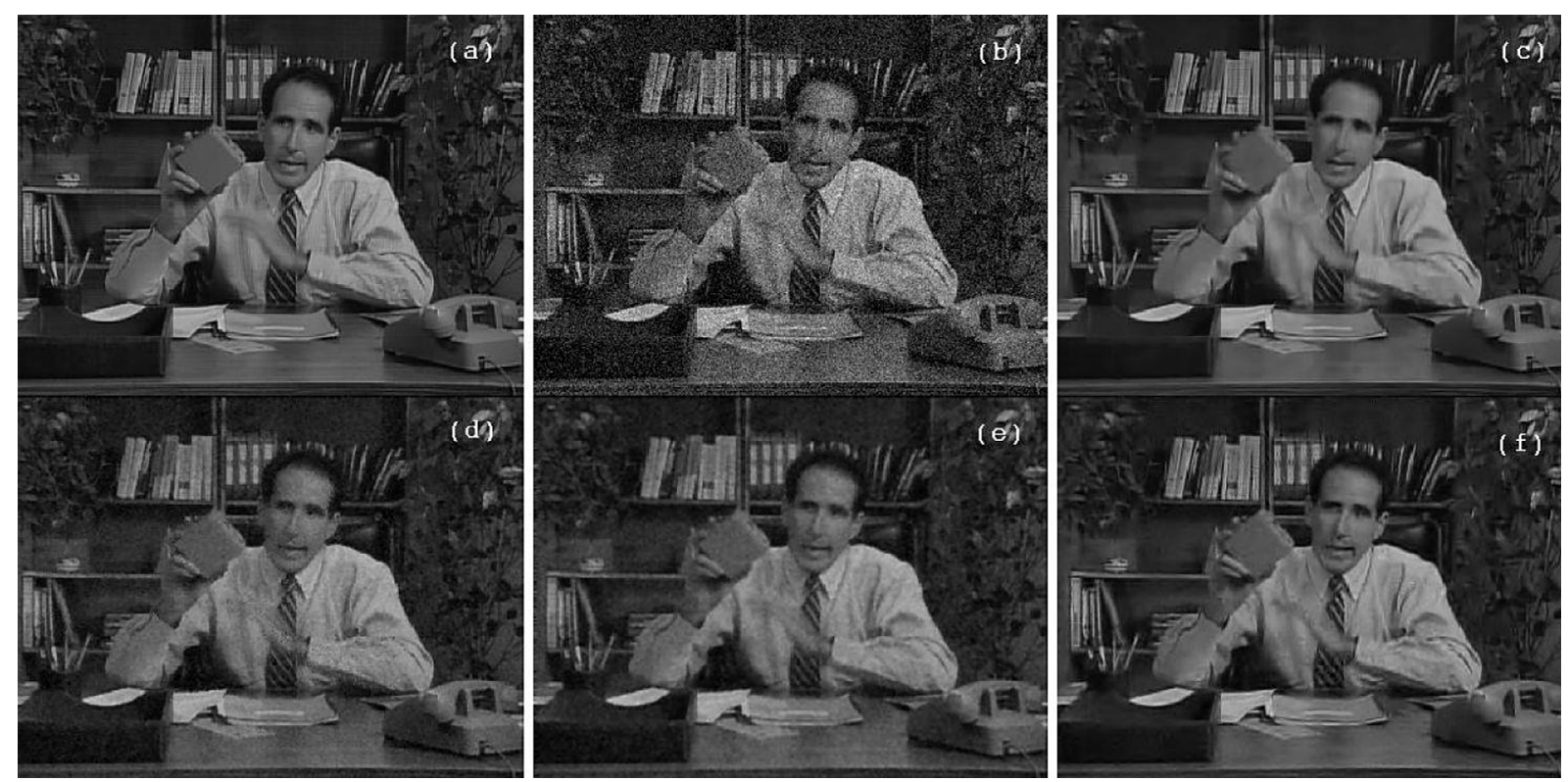

Fig. 2. "Salesman" Sequence (a) original frame, (b) Frame with additive Gaussian noise of $\sigma=20$. The filtered noisy frame with: (c) NNBA, (d) 3DKNN, (e) DnCNN and (f) VBM4D. 

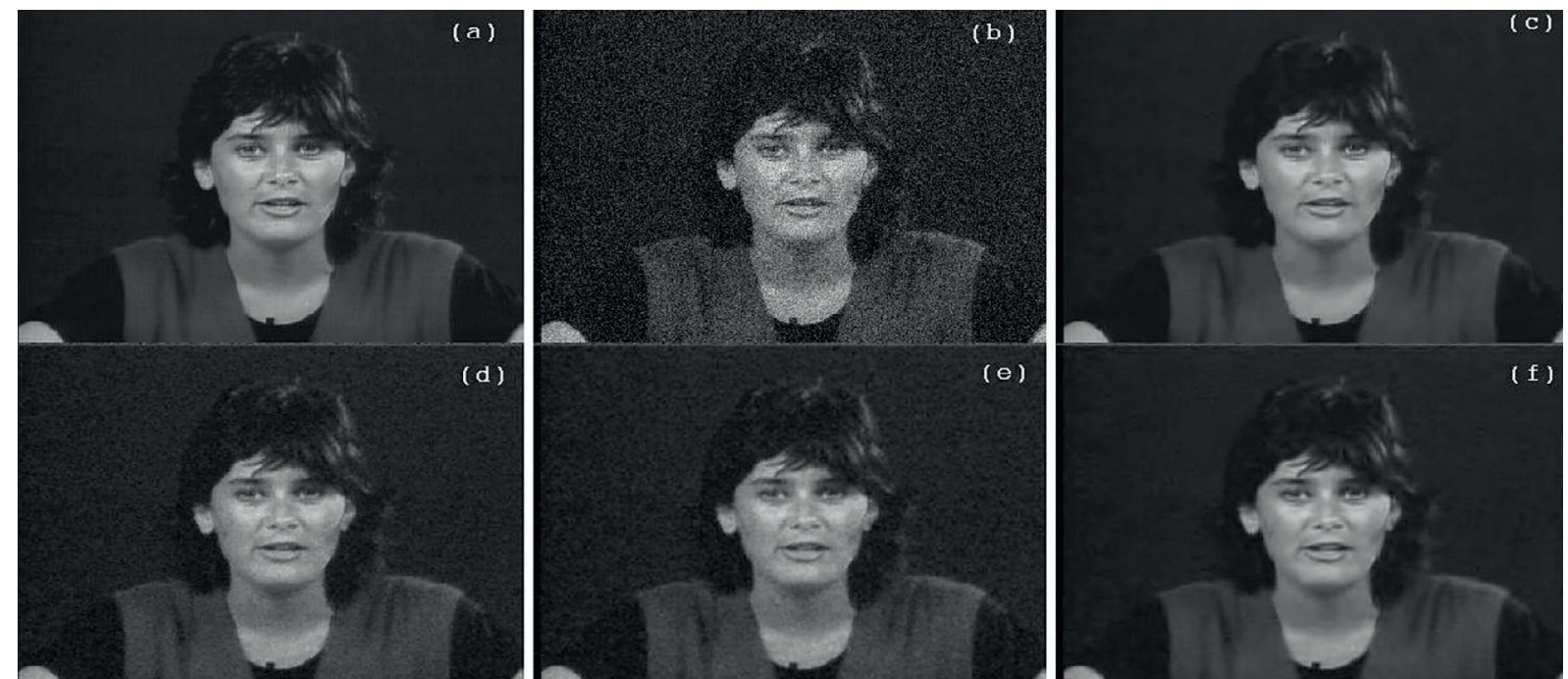

Fig. 3. "Miss America" Sequence. (a) Original frame (b) frame with additive Gaussian noise of $\sigma=20$. The filtered noisy frame with: (c) NNBA, (d) 3DKNN, (e) DnCNN and (f) VBM4D

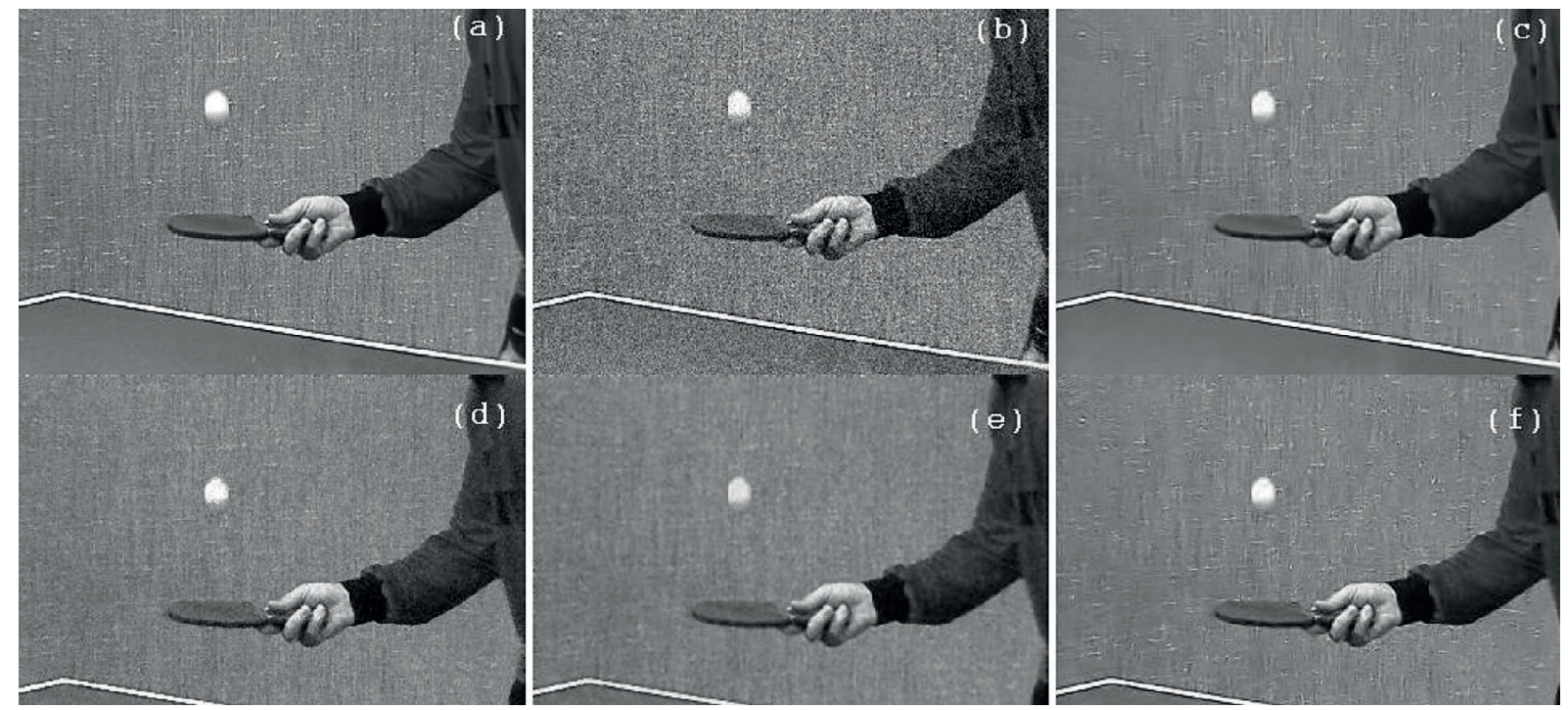

Fig. 4. "Tennis" Sequence. (a) Original frame. (b) Frame with additive Gaussian noise of $\sigma=$ 20. The filtered noisy frame with: (c) NNBA, (d) 3DKNN, (e) DnCNN and (f) VBM4D.
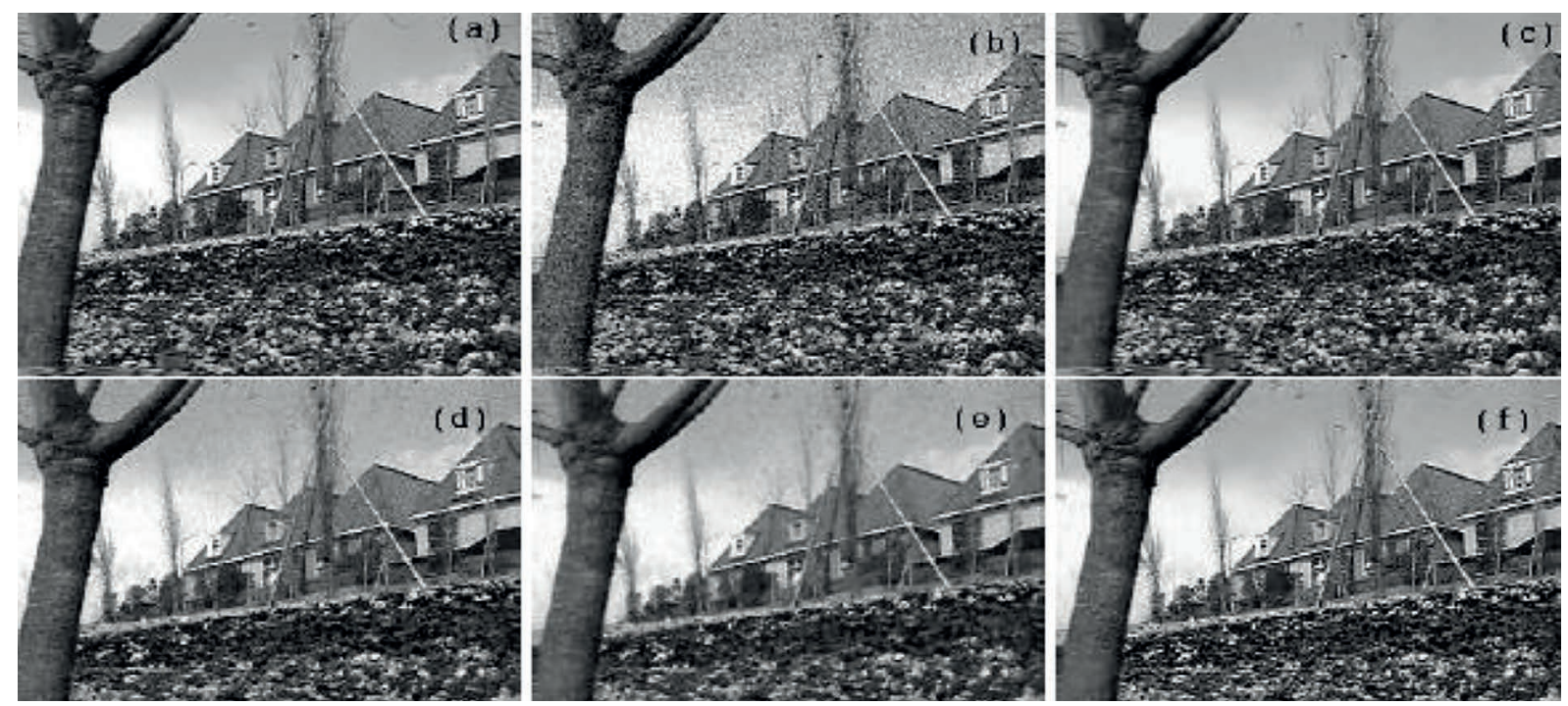

Fig. 5. "Flower Garden" Sequence. (a) original frame. (b) frame with additive Gaussian noise $\sigma$ $=15$. The level noisy frame with: (c) NNBA, (d) 3DKNN, (e) DnCNN and (f) VBM4D. 

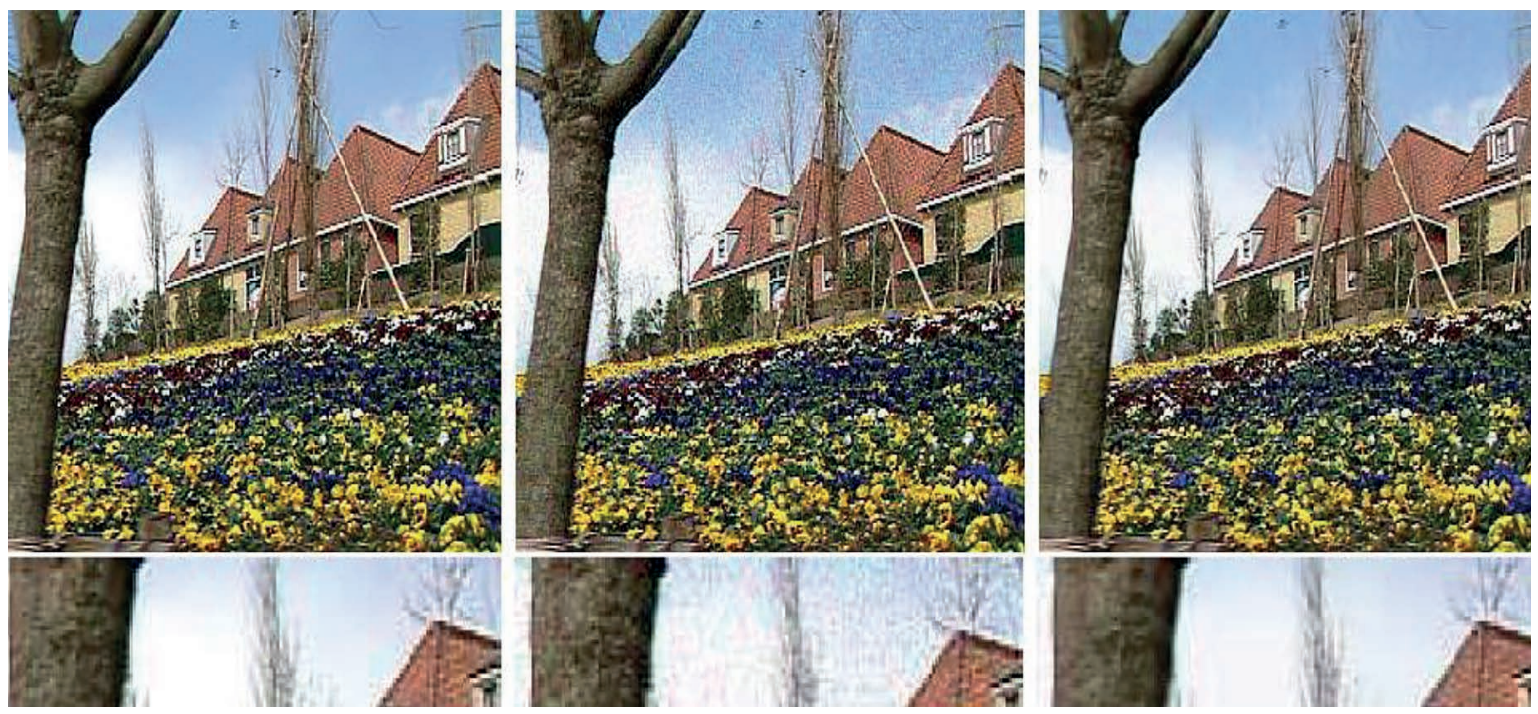

Fig. 6. "Flower Garden" color sequence: Top left original frame. Top middle: frame with additive Gaussian noise of $\sigma=15$. Top right: The filtered noisy frame with the proposed filtering algorithm. An expanded view of the initial noisy and processed picture, from left to right.
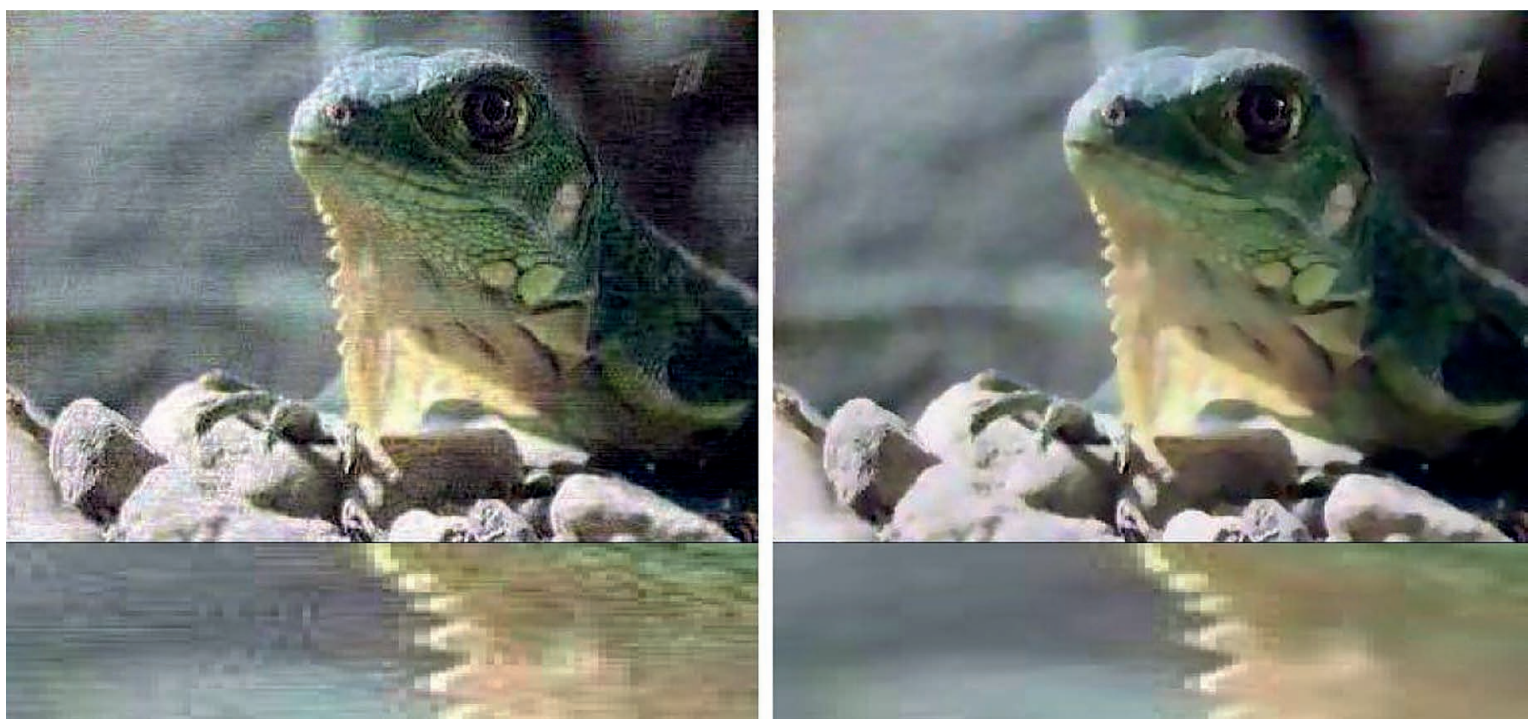

Fig. 7. "Lizard" sequence: Original frame . An expanded view of the noisy frame. The filtered frame by the proposed method. an expended view of the filtered frame.
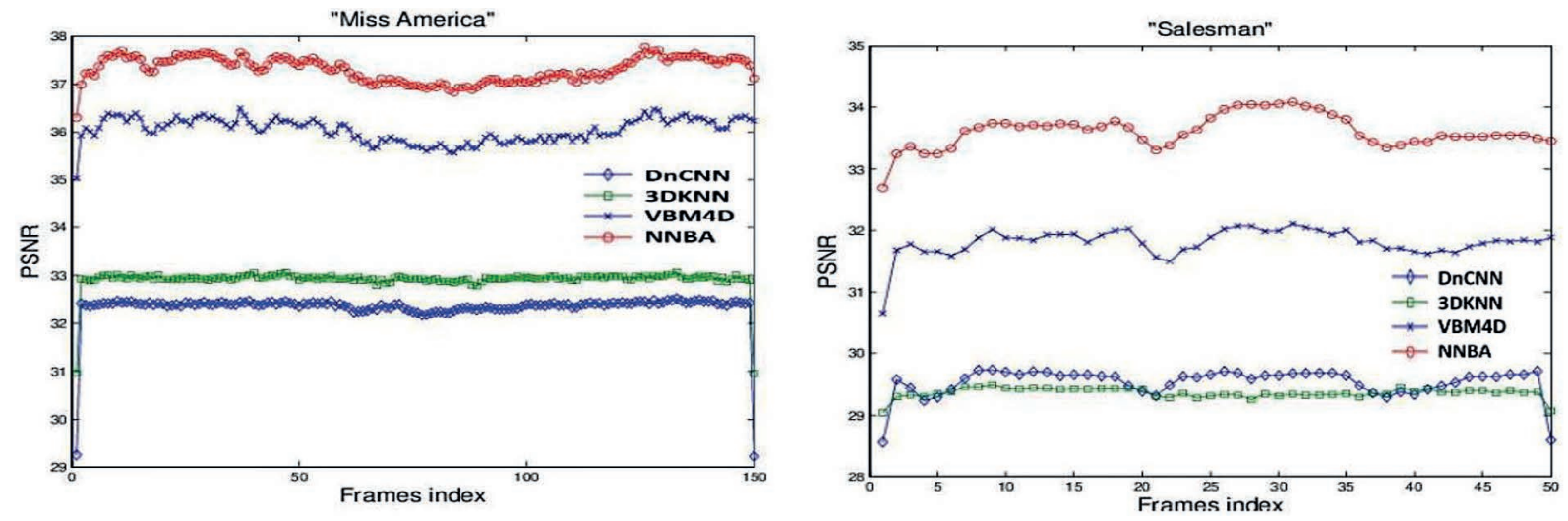

Fig. 8. PSNR versus index frame for filtered" Miss America" and" Salesman" sequences, with added noise with noise level 15. 

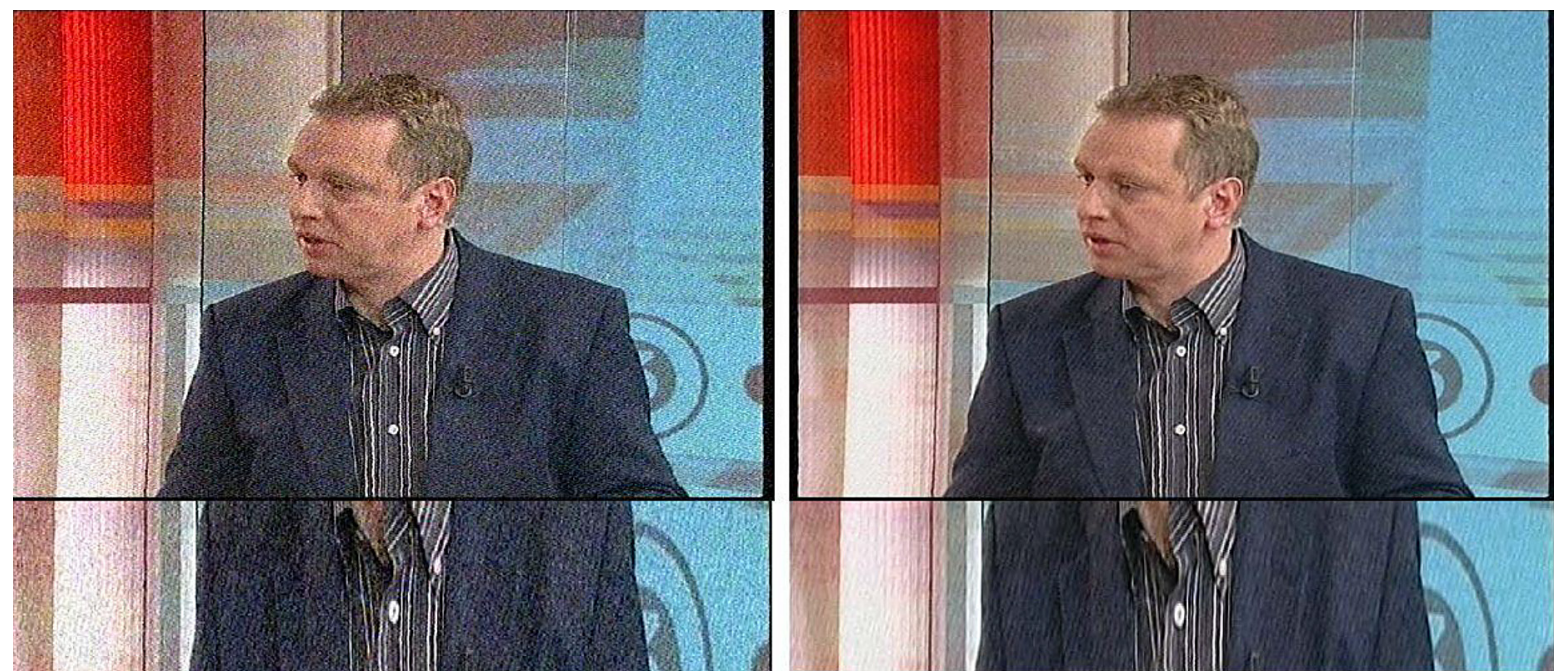

Fig. 9. "TV" sequence. The filtered frame by the proposed method. Lower right: An expended view of the noisy frame.

\section{CONCLUSION}

This paper proposes a new nonlinear Bayesian framework for noise reduction in video sequences. The proposed filter is developed from a variation problem that integrates a Bayesian term with a prior probability distribution of the observed noisy video sequence. The resultant formulation includes a nonlinear diffusion component that preserves critical features such as edges and corners. To exploit the noise redundancy, we constructed the Bayesian nonlinear filter on a block-wise non-local space-time region, which enables us to use intrinsic Bayesian filtering.

\section{REFERENCES}

1. Yahya A.A. Tan, J. Li L. Video noise reduction method using adaptive spatial-temporal filtering, Discrete Dyn. Nat. Soc. 2015; 10. doi: $10.1155 / 2015 / 351763$

2. Chatati S.M. Christopher S. A review on video noise reduction methods. International Conference on Recent Advances in Energy-efficient Computing and Communication (ICRAECC). 2019:1- 6. doi:10.1109/ICRAECC43874.2019.8995148.

3. Yan L., Yanfeng Q.Novel adaptive temporal filter based on motion compensation for video noise reduction, International Symposium on Communications and Information Technologies. 2006:10311034. doi:10.1109/ISCIT.2006.339934.

4. Rakhshanfar M., Amer A. Motion blur resistant method for temporal video noise reduction, IEEE International Conference on Image Pro- cessing (ICIP). 2014: 2694 -2698. doi:10.1109/ ICIP.2014.7025545.

5. Zuo C., Liu Y., Tan X., Wang W., Zhang, M. Video noise reduction based on a spatiotemporal kalmanbilateral mixture model, Sci. World J. 2013:438147. doi:10.1155/2013/438147.

6. Hong-Zhi W., Ling C., Shu-Liang X. Improved video noise reduction algorithm based on spatialtemporal combination. International Conference on Image and Graphics. 2013: 64-67. doi:10.1109/ ICIG.2013.19.

7. Mikula K., Preusser T., Rumpf M. Morphological image sequence processing, Comput. Vis. Sci.2004, 6 (4): 197-209. doi : 10.1007/s00791004-0129-0

8. Perona P. Malik J. Scale-space and edge detection using anisotropic diffusion, IEEE Trans. Pattern Anal. Mach. Intell.1990, 12 (7):629-639. doi:10.1109/34.56205

9. Lee S. H., Kang M. G. Space-time video filtering algorithm based on 3-d anisotropic diffusion equation. International Conference on Image Processing. ICIP98, 1998, 2: 447-450. doi:10.1109/ ICIP.1998.723418

10. Jahne J.B. Computer Vision and Applications: A Guide for Students and Practitioners, 2007, Elsevier.

11. Bovik A.C. Handbook of Image and Video Processing (Communications, Networking and Multimedia), 2005, Academic Press, Inc., USA.

12. Scharr H., Spies H. Accurate optical flow in noisy image sequences using flow adapted anisotropic diffusion, Signal Pross. 2005, 20(6): 537-553. doi:10.1016/j.image.2005.03.005

13. Hadj Fredj A., Malek J, Gpu-based anisotropic diffusion algorithm for video image noise reduction, Microprocessors and Microsystems. 2017,53: 190- 
201. doi: 10.1016/j.micpro.2017.08.003

14. Zhang K, Zuo W., Chen Y., Meng D., Zhang L. Beyond a Gaussian Denoiser: Residual Learning of Deep CNN for Image Denoising, IEEE Trans. Image Process. 2017; 26 (7): 3142-3155, doi:10.1109/ TIP.2017.2662206

15. Gorjizadeh S., Sadegh P., Siavash A. Noisy image segmentation using a self -organizing map network. Adv. Sci. Technol. Res. J. 2015; 9(26):118-123.

16. Davy A., Ehret T., Morel J.-M., Arias, P. Facciolo, G. Video noise reduction by combining patch search and cnns, Comput. Vis. Sci. 2021; 63: 7388. doi:10.1007/s10851-020-00995-0

17. Monteil J, Beghdadi A. A new interpretation and improvement of the nonlinear anisotropic diffusion for image enhancement, IEEE Trans. Pattern Anal. Mach. Intell. 1999; 21 (9): 940-946, doi: 10.1109/34.790435

18. Oksendal B. Stochastic Differential Equations: An Introduction with Applications. 2005; Springer.

19. Risken H. The Fokker-Planck Equation: Methods of Solutions and Applications, Springer Series in Synergetics, 1996; Springer.

20. Lebrun M., Buades A., More J.-M. A nonlocal bayesian image noise reduction algorithm, Siam J. Imaging Sci. 2013; 6 (3):1665-1688, doi: 10.5201/ ipol.2013.16.

21. Dabov K., Foi A., Katkovnik V., Egiazarian K.Image processing: Algorithms and systems, neural networks, and machine learning, in: Image noise reduction with block-matching and 3D filtering. 2006; 6064: 354 - 365, doi:10.1109/ ICIP.2014.7025545

22. Kostadin D., Foi A., Egiazarian K. Video noise reduction by sparse $3 \mathrm{~d}$ transform-domain collaborative filtering, 15th European Signal Processing
Conference. 2007: 145-149.

23. Maggioni M., Boracchi G., Foi A., Egiazarian K. Video noise reduction deblocking and enhancement through separable 4-d nonlocal spatiotemporal transforms, IEEE Tran. Image Proc. 2015; 21 (9): 39523966. doi: 10.1109/TIP.2012.2199324

24. Maggioni, F. VB4D open source (2021. [Online].). URL http://www.cs.tut.fi/ foi/GCF-BM3D/

25. Zlokolica V., Philips W. Motion- and detail-adaptive noise reduction of video, in: E. R. Dougherty, J. T. Astola, K. O. Egiazarian (Eds.), Image Processing: Algorithms and Systems III, Vol. 5298, International Society for Optics and Photonics, SPIE, 2004: 403412, doi:10.1117/12.520847

26. Maggioni M., Sanchez-Monge E.,'Foi, A. Joint removal of random and fixed-pattern noise through spatiotemporal video filtering, IEEE Trans. Image Process. 2014; 23 (10): 4282-4296. doi:10.1109/ TIP.2014.2345261.

27. Hsia S.-C., Hsu W.-C., Tsai C.-L. High-efficiency tv video noise reduction through adaptive spatialtemporal frame filtering, J. Real Time Image Proc. 2015; 10(3): 561-572. doi: 10.1155/2013/438147.

28. Almahdi R., Hardie R. C. Recursive non-local means filter for video noise reduction, J. Image Video Proc. 2017, 29. doi:10.1186/s13640017-0177-2.

29. Arias P., Morel J.-M. Video noise reduction via empirical Bayesian estimation of space-time patches, J. Math. Imaging Vis. 2018 ; 60: 70-93. doi :10.1007/s10851-017-0742-4

30. Uttenweiler D., Weber C., Jahne B., Fink R. H. A. Spatiotemporal" anisotropic diffusion filtering to improve signal-to-noise ratios and object restoration in fluorescence microscopic image sequences. J. Biomed. Opt. 2005 ; 8 (1): 40-47. doi: $10.1117 / 1.1527627$ 